\title{
The acute effects of strength, endurance and concurrent exercises on the Akt/mTOR/p70 6 S6K1 and AMPK signaling pathway responses in rat skeletal muscle
}

\author{
E.O. de Souza ${ }^{1}$, V. Tricoli $^{1}$, C. Bueno Junior ${ }^{2}$, M.G. Pereira ${ }^{3}$, P.C. Brum ${ }^{1}$, E.M. Oliveira ${ }^{1}$, \\ H. Roschel ${ }^{1}$, M.S. Aoki ${ }^{4}$ and C. Urginowitsch ${ }^{1}$ \\ ${ }^{1}$ Escola de Educação Física e Esporte, Universidade de São Paulo, São Paulo, SP, Brasil \\ ${ }^{2}$ Escola de Educação Física e Esporte de Ribeirão Preto, Universidade de São Paulo, Ribeirão Preto, SP, Brasil \\ ${ }^{3}$ Departamento de Anatomia, Instituto de Ciências Biomédicas, Universidade de São Paulo, São Paulo, SP, Brasil \\ ${ }^{4}$ Escola de Artes, Ciências e Humanidades, Universidade de São Paulo, São Paulo, SP, Brasil
}

\begin{abstract}
The activation of competing intracellular pathways has been proposed to explain the reduced training adaptations after concurrent strength and endurance exercises (CE). The present study investigated the acute effects of CE, strength exercises $(\mathrm{SE})$, and endurance exercises (EE) on phosphorylated/total ratios of selected AMPK and Akt/mTOR/p70 ${ }^{\mathrm{S} 6 \mathrm{~K} 1}$ pathway proteins in rats. Six animals per exercise group were killed immediately $(0 \mathrm{~h})$ and $2 \mathrm{~h}$ after each exercise mode. In addition, 6 animals in a non-exercised condition (NE) were killed on the same day and under the same conditions. The levels of AMPK, phospho-Thr ${ }^{172}$ AMPK (p-AMPK), Akt, phospho-Ser ${ }^{473}$ Akt (p-Akt), p70 ${ }^{\mathrm{S} 6 \mathrm{~K} 1}$, phospho-Thr ${ }^{389}-\mathrm{p} 70^{\mathrm{S} 6 \mathrm{~K} 1}$ (p-p70 ${ }^{\mathrm{S} 6 \mathrm{~K} 1}$ ), mTOR, phospho-Ser ${ }^{2448}$ mTOR (p-mTOR), and phospho-Thr ${ }^{1462}$-TSC2 ( $p$-TSC2) expression were evaluated by immunoblotting in total plantaris muscle extracts. The only significant difference detected was an increase (i.e., $87 \%$ ) in Akt phosphorylated/total ratio in the CE group $2 \mathrm{~h}$ after exercise compared to the NE group $(P=0.002)$. There were no changes in AMPK, TSC2, mTOR, or $\mathrm{p} 70^{\mathrm{s} 6 \mathrm{~K} 1}$ ratios when the exercise modes were compared to the NE condition $(P \geqslant 0.05)$. In conclusion, our data suggest that low-intensity and low-volume CE might not blunt the training-induced adaptations, since it did not activate competing intracellular pathways in an acute bout of strength and endurance exercises in rat skeletal muscle.
\end{abstract}

Key words: Strength exercise; Concurrent training; Cell signaling; AMPK

\section{Introduction}

The combination of strength and endurance exercises throughout a training period is referred to as concurrent training or concurrent exercise (CE) $(1,2)$. The CE mode is thought to hamper strength-training induced skeletal muscle adaptations (i.e., reduced muscle strength and muscle fiber hypertrophy) when compared to strength training alone. This diminished training adaptation is known as the interference effect $(3,4)$. Among the several theories postulated to explain the interference effect $(2,3,5)$, a molecular hypothesis has been suggested to explain the reduced training adaptations after CE. This hypothesis claims that CE may simultaneously activate competing intracellular pathways that may down-regulate the adaptive response of muscle growth $(6,7)$.
In this regard, it has been demonstrated that strength exercise (SE) is capable of increasing the activation of the Akt/mTOR/p70 $6{ }^{\text {S61 } 1}$ pathway, and, as a consequence, muscle protein synthesis and accretion $(8,9)$. On the other hand, endurance exercise (EE) promotes changes in the mitochondrial biogenesis machinery through the activation of the AMPK-PGC-1 $\alpha$ pathway $(10,11)$. Accordingly, Atherton et al. (12) mimicked both EE and SE by applying either low- (LFS) or high-frequency (HFS) electro-stimulation, respectively, to scrutinize the activation of the aforementioned signaling pathways in rat skeletal muscle. The most important findings of the cited study were that the activation of the Akt/mTOR/p70 $36 \mathrm{~K} 1$ pathway and myofibrillar protein synthesis rate increased after HFS, while the AMPK-PGC- $1 \alpha$ pathway was activated only after LFS.

Correspondence: E.O. de Souza, Escola de Educação Física e Esporte, USP, Av. Prof. Mello Moraes, 65, 05508-030 São Paulo, SP, Brasil. E-mail: desouza.eo@gmail.com 
The phosphorylation of AMPK ( $p-A M P K)$ seems to trigger the interference effect at the molecular level (7). Once activated, p-AMPK inhibits the cell anabolic processes that demand ATP expenditure, such as protein synthesis, in order to replenish ATP stores $(13,14)$. Lower protein synthesis could be mediated in part by an increase in p-AMPK and a concomitant decrease in the anabolic response downstream of mTOR signaling (13).

Therefore, it seems reasonable to suggest that CE may increase AMPK activity, thus possibly attenuating mTOR phosphorylation and its downstream targets such as p70 6 K61 $(13,14)$. Thus, the purpose of the present study was to investigate the acute effects of EE, SE, and CE on selected AMPK and Akt/mTOR/p70 ${ }^{\text {S6K1 } 1}$ pathway proteins.

\section{Material and Methods}

\section{Experimental design}

Initially, the animals were acquainted with the handling and exercise procedures and then performed two functional tests: a graded treadmill exercise test (GTET) and one repetition maximum test (1RM). After the functional tests, 42 male Wistar rats $(345.3 \pm 27.1 \mathrm{~g}$ and 14 weeks old) were randomly assigned to four experimental groups: no exercise (i.e., sedentary control, NE, $n=6$ ); SE, $n=$ 12; EE, $n=12$, and $C E, n=12$. Animals in the exercise groups were killed immediately $(0 \mathrm{~h}, \mathrm{n}=6$ for each exercised group) and $2 \mathrm{~h}$ ( $\mathrm{n}=6$ for each exercised groups) after the completion of the exercise protocol. The NE animals were killed on the same day in random order and at the same time and conditions as the animals of the experimental groups. The plantaris muscle was excised from the animals and stored at $-80^{\circ} \mathrm{C}$ for further analysis.

\section{Animal care}

All animals were maintained on a $12: 12$-h dark-light cycle in a temperature-controlled environment $\left(22^{\circ} \mathrm{C}\right)$ with free access to standard laboratory chow (Nuvital Nutrientes S/A, Brazil) and tap water. The study was conducted according to the guidelines of the Brazilian College of Animal Experimentation (www.cobea.org.br). In addition, all procedures were approved by the Ethics Review Board of the Escola de Educação Física e Esporte, Universidade de São Paulo.

\section{Familiarization procedures}

Before the allocation to their respective groups, all animals were handled in their cages for 5 days to minimize contact stress. In addition, they underwent three familiarization sessions, separated by 48 -h intervals, to get acquainted with the exercise protocols (i.e., endurance and strength exercises).

\section{Functional tests}

GTET. Briefly, rats were placed in the treadmill and allowed to acclimatize for at least $30 \mathrm{~min}$. The GTET was then started at $0 \%$ grade and $6 \mathrm{~m} / \mathrm{min}$ with increments of $3 \mathrm{~m} / \mathrm{min}$ every $3 \mathrm{~min}$ until exhaustion. The maximum velocity (V.max) attained during the test was used to determine the training load (15).

$1 R M$ test. This test was performed in a strength training apparatus that was built exactly as described by Tamaki et al. (16). Animals were fitted with a canvas jacket able to limit twisting and flexion of their torsos and were suspended in the standard hindlimb position. Electrical stimulation (10-15 V, 0.3-s duration, 3-s intervals) was applied to the rat tail through a surface electrode. As a result, the rats extended their legs repeatedly, which lifted the weight-arm of the training apparatus. The load used to start the test was the animal's body mass. Then, animals were allowed up to five attempts to achieve the 1RM load with a 3-min interval between attempts. During the rest intervals, animals were returned to their cage. The maximum exercise load was taken to be when the animals were unable to move up the weight-arm of the training apparatus after an electrical stimulation.

\section{Exercise protocols}

Animals in the SE group performed five sets of 10 repetitions of the strength exercise with a load corresponding to $75 \%$ of the 1RM (i.e., $540.0 \pm 56.6 \mathrm{~g}$ ). Repetitions within sets were performed at 2-s intervals. A 2 -min interval was allowed between sets. Animals were returned to their cage during the rest interval between sets. EE animals ran for $60 \mathrm{~min}$ at $60 \%$ V.max (i.e., 11.43 $\pm 1.53 \mathrm{~m} / \mathrm{min}$ ), achieved during the GTET. Finally, CE animals performed both SE and EE protocols (i.e., $530.0 \pm 97.5 \mathrm{~g}$ and $12.6 \pm 2.4 \mathrm{~m} / \mathrm{min}$, respectively). Animals in the CE group performed the EE protocol first. Immediately after the completion of the EE bout, they were placed in the strength-training apparatus to perform the SE protocol.

\section{Sample analyses}

Animals were killed by decapitation immediately $(0 \mathrm{~h})$ and $2 \mathrm{~h}$ after the exercise protocols. In addition, the animals in the NE group $(n=6)$ were killed on the same day and under the same conditions. The plantaris muscles were removed from both legs, weighed on an analytical balance, frozen in liquid nitrogen, and stored at $-80^{\circ} \mathrm{C}$ for further analysis. To avoid differences in the time of death, the test order was counterbalanced among the groups.

\section{Immunoblotting}

Immunoblots of the plantaris muscle homogenates were performed as previously described (17). Briefly, tissues frozen in liquid nitrogen were homogenized in a buffer containing $1 \mathrm{mM}$ EDTA, $1 \mathrm{mM}$ EGTA, $2 \mathrm{mM}$ $\mathrm{MgCl}_{2}, 5 \mathrm{mM} \mathrm{KCl}, 25 \mathrm{mM}$ HEPES, pH 7.5, $100 \mu \mathrm{M}$ PMSF, $2 \mathrm{mM}$ DTT, 1\% Triton $\mathrm{X}-100$, and a protease inhibitor cocktail (P8340; 1:100, Sigma-Aldrich, USA). The 
samples were then subjected to SDS-PAGE in polyacrylamide gels $(6-15 \%)$ depending upon protein molecular weight. After electrophoresis, proteins were electrotransferred to nitrocellulose membranes (BioRad Biosciences, USA). Equal gel loading and transfer efficiency were monitored using $0.5 \%$ Ponceau $S$ staining of the blot membrane. The blotted membrane was then blocked $(5 \%$ BSA, $10 \mathrm{mM}$ Tris- $\mathrm{HCl}, \mathrm{pH} 7.6,150 \mathrm{mM} \mathrm{NaCl}$, and $0.1 \%$ Tween 20) for $2 \mathrm{~h}$ at room temperature and then incubated overnight at $4^{\circ} \mathrm{C}$ with specific antibodies against AMPK, phospho-Thr ${ }^{172}$ AMPK (p-AMPK), Akt, phospho-Ser ${ }^{473} A k t$ (p-Akt), p70 ${ }^{\text {S6K1 }}$, phospho-Thr ${ }^{389}$ p70 ${ }^{\text {S6K1 }}\left(p-p 70^{\text {S6K1 }}\right)$, mTOR, phospho-Ser ${ }^{2448}$ mTOR ( $p-$ mTOR), and phospho-Thr ${ }^{1462}$-TSC2 (p-TSC2) (Cell Signaling Technology, USA). Binding of one antibody per gel was detected with the use of peroxidaseconjugated secondary antibodies (rabbit or mouse, depending on the protein, for $2 \mathrm{~h}$ at room temperature) and developed using enhanced chemiluminescence (Amersham Biosciences, USA) detected by autoradiography. Quantification analysis of the blots was performed using the Image $\mathrm{J}$ software (Image $\mathrm{J}$ based on $\mathrm{NIH}$ image), which measures pixel density of the autoradiographies. Targeted bands were normalized to $\alpha$-tubulin antibody (1:1000; Santa Cruz Biotechnology, USA). The phosphorylated/total $(\mathrm{P} / \mathrm{T})$ ratios were calculated to assess changes in the activity of these proteins.

\section{Statistical analysis}

After normality (i.e., Shapiro Wilk test) and variance assurance (i.e., Levene test), treatments were compared by two-way analysis of variance, assuming time (i.e., 0 and $2 \mathrm{~h}$ ) and group (i.e., exercise conditions) as factors. Whenever a significant F-value was obtained, the Tukey post hoc test was applied for multiple comparison purposes. The level of significance was set at $P<0.05$. Data are reported as means \pm SE.

\section{Results}

\section{Functional tests}

There were no differences in the $1 \mathrm{RM}$ test $(\mathrm{F}=1.74$; $P \geqslant 0.21)$ or graded treadmill exercise test $(F=1.08 ; P \geqslant$ 0.38 ) between groups at any time.

\section{Molecular responses}

Since the total protein content remained unchanged between groups and times, data are presented as $\mathrm{P} / \mathrm{T}$ ratios to assess changes in the phosphorylation of the selected proteins due to the exercise protocols.

The Akt P/T ratio was significantly higher (i.e., $87 \%$ ) in the CE group at the 2-h time point when compared to the NE group $(P=0.002)$. There were no differences in the AMPK, TSC2, mTOR, or $\mathrm{p} 70^{\mathrm{S} 6 \mathrm{~K} 1} \mathrm{P} / \mathrm{T}$ ratios between groups $(P \geqslant 0.05$; Figure 1$)$, indicating no effect of the exercise bouts (i.e., EE, SE and CE) on the activity of the selected proteins across time.

\section{Discussion}

The purpose of this study was to investigate the acute effects of EE, SE, and CE on selected AMPK and Akt/ $\mathrm{mTOR} / \mathrm{p} 70^{\mathrm{S} 6 \mathrm{~K} 1}$ pathway proteins. We did not obtain any data to support the notion that CE might activate the AMPK pathway and, as a consequence, inhibit the Akt/ $\mathrm{mTOR} / \mathrm{p} 70^{\mathrm{S} 6 \mathrm{~K} 1}$ signaling pathway. The only significant difference detected was the increase (i.e., $87 \%$ ) in the Akt $P / T$ ratio in the CE group $2 \mathrm{~h}$ after exercise $(P=0.002)$.

The strength exercise used in the present study was developed by Tamaki et al. (16), and has been shown to increase skeletal muscle mass in rats. Tamaki et al. reported a $31.4 \%$ increase in muscle weight/body mass ratio after 12 weeks of training. However, Tamaki's exercise mode does not optimize the eccentric phase of the lifts. In humans, maximal eccentric contractions are reported to be effective in stimulating $\mathrm{mTOR}$ and $\mathrm{p} 70^{\mathrm{S} 6 \mathrm{~K} 1}$ via an Aktindependent pathway $(18,19)$. This effect may be mediated by a combination of greater tension and stretching of the skeletal muscles during the descending phase of the lift. In the current study, the animal does not need to control the descending part of the squatting movement, possibly decreasing the mechanical load generated during the eccentric phase. Therefore, in an acute condition, this strength exercise may not produce sufficient mechanical stress to activate the Akt/mTOR/p70 $66 \mathrm{~K} 1$ signaling pathway. Moreover, the absence of activation of the selected proteins might be associated with the exercise bout intensity. In fact, the animals in the present study exercised with lower intensity protocols compared with previous studies with the same weight-lifting model $(16,20)$. Importantly, although one may speculate that this was due to the low number of familiarization sessions employed (three), we chose this approach in order to avoid any training effects that could have occurred due to a longer familiarization period, which could have potentially blunted the muscle response to the exercise protocol.

Furthermore, we attempted to reproduce a typical CE bout used by humans in order to investigate the acute responses of the aforementioned pathways. AMPK is an important protein kinase involved in the control of the cell energy status that seems to be activated by either a strength or endurance exercise bout $(10,21)$. The activation of AMPK reduces skeletal muscle protein synthesis by inhibiting the mTOR signaling pathway via activation of tuberous sclerosis complex 2 (TSC2) (13). We found neither a significant activation of AMPK and TSC2 nor a down-regulation of mTOR and p70 $56 \mathrm{~K} 1$ after CE. Predominantly, controlled animal conditions have shown AMPK activation followed by a down-regulation in proteins involved in mRNA translation $(13,14)$. For example, Thomson et al. (14) reported an acute attenuation in $\mathrm{p}-\mathrm{p} 70^{\mathrm{S6K} 1 / \mathrm{Thr} 389}, 4 \mathrm{E}-\mathrm{BP} 1$ and eEF2 ${ }^{\text {Thr56 }}$ after AMPK activation. However, they applied electrically 

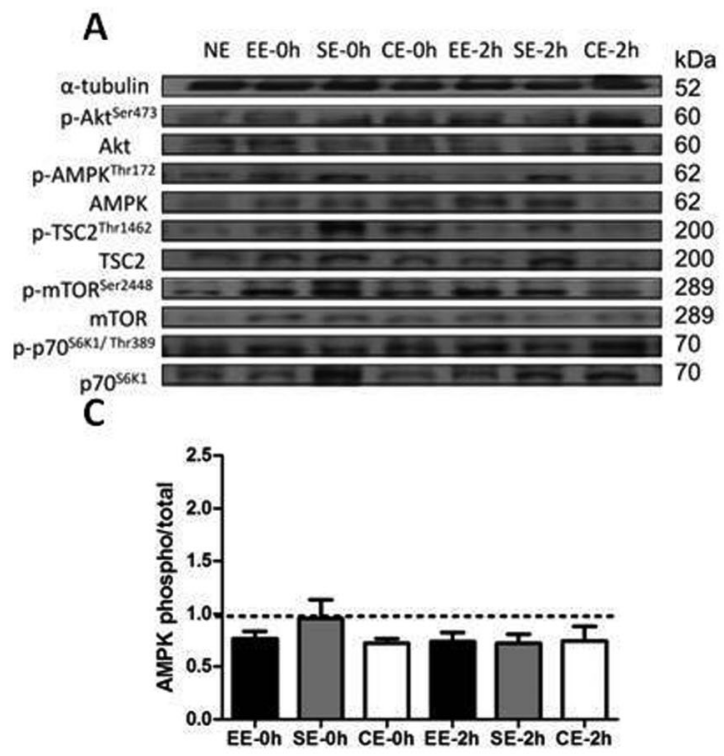

E

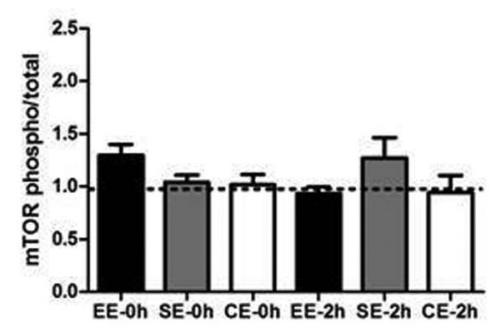

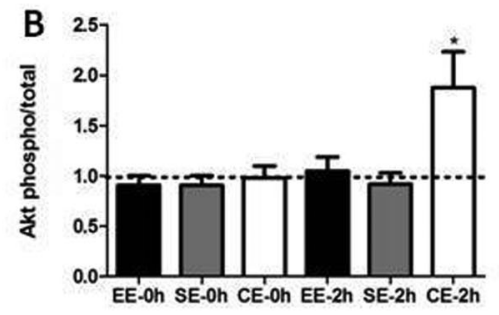
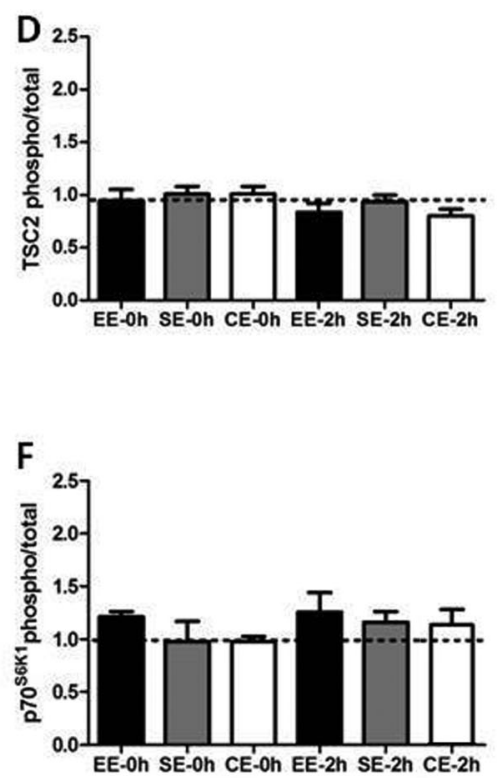

Figure 1. Phosphorylated/total ratio Akt, AMPK, TSC2, $m T O R$, and $p 70^{\mathrm{S} 6 \mathrm{~K} 1}$ protein expression for endurance exercise $(\mathrm{EE} ; \mathrm{n}=6)$, strength exercise (SE; $n=6$ ), and concurrent exercise (CE; $n=6$ ) groups immediately after $(0 \mathrm{~h})$ and $2 \mathrm{~h}$ after exercise protocols. The traced line refers to the no exercise control group (NE; $n=6$ ) (means $\pm S E$ ). ${ }^{*}=0.002$ compared to the NE group [two-way ANOVA (F test $=4.406)$ ].

stimulated lengthening muscle contractions, which were produced through high-frequency stimulation of the sciatic nerve. In addition, 40 min before the muscle stimulation, rats were subcutaneously injected with AICAR, an AMPK agonist. On the other hand, our exercise protocols were devoid of pharmacological interventions or electrically elicited muscle contractions. Furthermore, an increase in p-AMPK via exercise only (i.e., non-pharmacologically induced or induced by electrical stimulation) seems to be dependent on chronic stimulation at greater exercise intensities. In this regard, Durante et al. (10) found detectable differences in AMPK activity in male SpragueDawley rats after a 4-week endurance training protocol ranging from 16 to $31 \mathrm{~m} / \mathrm{min}$ at a $15 \%$ grade. Thus, our data suggest that it might be difficult to mimic an acute $C E$ condition that is adequate to investigate the proposed hypothesis of an interference effect between AMPK and Akt/ $\mathrm{mTOR} / \mathrm{p} 70^{\text {S6K1 }}$ pathways in animals.

Regarding the significant difference detected in the Akt
$\mathrm{P} / \mathrm{T}$ ratio in the CE group $2 \mathrm{~h}$ after exercise, it is important to note that Akt activity has been demonstrated to be upregulated (7), down-regulated (22), or unchanged (23) in response to strength exercise bouts. As Akt might suffer a cross-talk from different cell signaling pathways and several stimuli (24), we suggest that the increased effect observed on Akt P/T after the CE protocol might be an overlap of both the endurance and the strength exercise stimuli as neither of the isolated exercise protocols produced a significant activation in the selected proteins.

We did not confirm that CE may simultaneously activate competing intracellular pathways in an acute bout of strength and endurance exercises in rat skeletal muscle. In addition, our data suggest that it might be difficult to create a concurrent training condition similar to a real-world training routine in an animal exercise mode. Future studies in human and animal models are required to confirm the molecular hypothesis that has been proposed to explain the CE-induced impaired training adaptations. 


\section{Acknowledgments}

Research supported by FAPESP (\#2007/02738-6 for
E.O. de Souza, and \#2010/51428-2 for H. Roschel) and CNPq (\#152658/2011-4 for E.O. de Souza and \#470207/ 2008-6 and \#303162/2008-2 for C. Urginowitsch).

\section{References}

1. Kraemer WJ, Patton JF, Gordon SE, Harman EA, Deschenes MR, Reynolds K, et al. Compatibility of high-intensity strength and endurance training on hormonal and skeletal muscle adaptations. J Appl Physiol 1995; 78: 976-989.

2. Leveritt M, Abernethy PJ, Barry BK, Logan PA. Concurrent strength and endurance training. A review. Sports Med 1999; 28: 413-427, doi: 10.2165/00007256-199928060-00004.

3. Docherty D, Sporer B. A proposed model for examining the interference phenomenon between concurrent aerobic and strength training. Sports Med 2000; 30: 385-394, doi: 10.2165/00007256-200030060-00001.

4. Hickson RC. Interference of strength development by simultaneously training for strength and endurance. Eur $J$ Appl Physiol Occup Physiol 1980; 45: 255-263, doi: 10.1007/BF00421333.

5. Leveritt M, Abernethy PJ, Barry B, Logan PA. Concurrent strength and endurance training: the influence of dependent variable selection. J Strength Cond Res 2003; 17: 503-508.

6. Coffey VG, Hawley JA. The molecular bases of training adaptation. Sports Med 2007; 37: 737-763, doi: 10.2165/ 00007256-200737090-00001.

7. Nader GA. Concurrent strength and endurance training: from molecules to man. Med Sci Sports Exerc 2006; 38: 1965-1970, doi: 10.1249/01.mss.0000233795.39282.33.

8. Kubica N, Bolster DR, Farrell PA, Kimball SR, Jefferson LS. Resistance exercise increases muscle protein synthesis and translation of eukaryotic initiation factor 2Bepsilon mRNA in a mammalian target of rapamycin-dependent manner. J Biol Chem 2005; 280: 7570-7580, doi: 10.1074/ jbc.M413732200.

9. Leger B, Cartoni R, Praz M, Lamon S, Deriaz O, Crettenand A, et al. Akt signalling through GSK-3beta, mTOR and Foxo1 is involved in human skeletal muscle hypertrophy and atrophy. J Physiol 2006; 576: 923-933, doi: 10.1113/ jphysiol.2006.116715.

10. Durante PE, Mustard KJ, Park SH, Winder WW, Hardie DG. Effects of endurance training on activity and expression of AMP-activated protein kinase isoforms in rat muscles. Am J Physiol Endocrinol Metab 2002; 283: E178-E186.

11. Norrbom J, Sundberg CJ, Ameln H, Kraus WE, Jansson E, Gustafsson T. PGC-1alpha mRNA expression is influenced by metabolic perturbation in exercising human skeletal muscle. J Appl Physiol 2004; 96: 189-194, doi: 10.1152/ japplphysiol.00765.2003.

12. Atherton PJ, Babraj J, Smith K, Singh J, Rennie MJ, Wackerhage $\mathrm{H}$. Selective activation of AMPK-PGC-1alpha or PKB-TSC2-mTOR signaling can explain specific adaptive responses to endurance or resistance training-like electrical muscle stimulation. FASEB J 2005; 19: 786-788.

13. Bolster DR, Crozier SJ, Kimball SR, Jefferson LS. AMPactivated protein kinase suppresses protein synthesis in rat skeletal muscle through down-regulated mammalian target of rapamycin (mTOR) signaling. J Biol Chem 2002; 277:
23977-23980, doi: 10.1074/jbc.C200171200.

14. Thomson DM, Fick CA, Gordon SE. AMPK activation attenuates S6K1, 4E-BP1, and eEF2 signaling responses to high-frequency electrically stimulated skeletal muscle contractions. J Appl Physiol 2008; 104: 625-632, doi: 10.1152/japplphysiol.00915.2007.

15. Pereira MG, Ferreira JC, Bueno CR Jr, Mattos KC, Rosa $\mathrm{KT}$, Irigoyen $\mathrm{MC}$, et al. Exercise training reduces cardiac angiotensin II levels and prevents cardiac dysfunction in a genetic model of sympathetic hyperactivity-induced heart failure in mice. Eur J Appl Physiol 2009; 105: 843-850, doi: 10.1007/s00421-008-0967-4

16. Tamaki T, Uchiyama S, Nakano S. A weight-lifting exercise model for inducing hypertrophy in the hindlimb muscles of rats. Med Sci Sports Exerc 1992; 24: 881-886.

17. Towbin H, Staehelin T, Gordon J. Electrophoretic transfer of proteins from polyacrylamide gels to nitrocellulose sheets: procedure and some applications. Proc Natl Acad Sci U S A 1979; 76: 4350-4354, doi: 10.1073/pnas.76.9.4350.

18. Eliasson J, Elfegoun T, Nilsson J, Kohnke R, Ekblom B, Blomstrand $\mathrm{E}$. Maximal lengthening contractions increase p70 S6 kinase phosphorylation in human skeletal muscle in the absence of nutritional supply. Am J Physiol Endocrinol Metab 2006; 291: E1197-E1205, doi: 10.1152/ ajpendo.00141.2006.

19. Roschel H, Ugrinowistch C, Barroso R, Batista MA, Souza $\mathrm{EO}$, Aoki MS, et al. Effect of eccentric exercise velocity on akt/mtor/p70(s6k) signaling in human skeletal muscle. Appl Physiol Nutr Metab 2011; 36: 283-290, doi: 10.1139/h10-111.

20. Tamaki T, Uchiyama S, Uchiyama Y, Akatsuka A, Yoshimura S, Roy RR, et al. Limited myogenic response to a single bout of weight-lifting exercise in old rats. Am J Physiol Cell Physiol 2000; 278: C1143-C1152.

21. Dreyer HC, Fujita S, Cadenas JG, Chinkes DL, Volpi E, Rasmussen BB. Resistance exercise increases AMPK activity and reduces $4 \mathrm{E}-\mathrm{BP} 1$ phosphorylation and protein synthesis in human skeletal muscle. J Physiol 2006; 576: 613-624, doi: 10.1113/jphysiol.2006.113175.

22. Deldicque L, Atherton $P$, Patel R, Theisen D, Nielens $H$, Rennie MJ, et al. Decrease in Akt/PKB signalling in human skeletal muscle by resistance exercise. Eur J Appl Physiol 2008; 104: 57-65, doi: 10.1007/s00421-008-0786-7.

23. Karagounis LG, Yaspelkis BB III, Reeder DW, Lancaster GI, Hawley JA, Coffey VG. Contraction-induced changes in TNFalpha and Akt-mediated signalling are associated with increased myofibrillar protein in rat skeletal muscle. Eur $J$ Appl Physiol 2010; 109: 839-848, doi: 10.1007/s00421-0101427-5.

24. Sartori R, Milan G, Patron M, Mammucari C, Blaauw B, Abraham R, et al. Smad2 and 3 transcription factors control muscle mass in adulthood. Am J Physiol Cell Physiol 2009; 296: C1248-C1257, doi: 10.1152/ajpcell.00104.2009. 